\title{
Wild transthyretin amyloid cardiomyopathy recognition with noninvasive strategies: A case report and diagnostic approach for transthyretin-related cardiomyopathy
}

\author{
Gaspar Del Rio-Pertuz MD, Leigh Ann Jenkins MD, Pooja Sethi MD, Erwin Argueta-Sosa MD
}

\begin{abstract}
Recent studies suggest that the prevalence of wild type transthyretin cardiomyopathy (wATTR-CM) is substantially higher than previously appreciated in older adults with heart failure. Advances in nuclear imaging using bone avid radiotracers permit diagnosis of transthyretinrelated cardiomyopathy (ATTR-CM) without a tissue biopsy. We describe two wATTR-CM cases that were diagnosed using noninvasive methods and demonstrate this diagnostic approach for transthyretin-related cardiomyopathy.
\end{abstract}

Keywords: amyloid, cardiomyopathy

\section{INTRODUCTION}

Amyloidosis is a disease that affects multiple organs, especially the central and peripheral nervous system, kidneys, and heart. ${ }^{1}$ Two types of amyloid infiltrate the heart: immunoglobulin light-chain (AL or primary systemic) amyloid and transthyretin amyloid (ATTR). Transthyretin-related cardiomyopathy (ATTR-CM) is an infiltrative progressive disease caused by the deposition of transthyretin proteins, synthesized in the liver, into the interstitial space of the myocardium that lead to dysfunction and stiffness. ${ }^{2,3}$ Transthyretin-related amyloidosis includes two forms. First, a familial disease arising from misfolding of mutated transthyretin called hereditary amyloid cardiomyopathy (hATTR-CM), and second, a sporadic non-genetic disease caused by the disaggregation of wild-type transthyretin. The latter is referred to as wild-type transthyretin cardiomyopathy (wATTR-CM), also known as senile systemic amyloidosis. ${ }^{4}$

Corresponding author: Gaspar Del-Rio-Pertuz Contact Information: Gaspar.Del-Rio-Pertuz@ttuhsc.edu DOI: 10.12746/swrccc.v10i42.999
Recent studies have suggested that the prevalence of WATTR-CM is substantially higher than previously appreciated in older adults with heart failure. This is mainly because advances in nuclear imaging, such as bone avid radiotracers, allow a noninvasive diagnosis of ATTR-CM (either hATTR-CM or wATTR$\mathrm{CM}$ ) without the need for a tissue biopsy. ${ }^{5}$ Here we describe two cases of wATTR-CM that were diagnosed using noninvasive methods. We also discussed a diagnostic approach for ATTR-CM based on the most recent literature.

\section{Cases}

Case 1: An 85-year-old man was being evaluated with atrial fibrillation that had recurred after direct current cardioversion. He was unable to tolerate beta-blockers and had lower extremity edema and mild dyspnea with minimal activity for the past three years. A transthoracic echocardiogram (TTE) demonstrated a left ventricular ejection fraction (LVEF) of $36 \%$, left ventricular end-diastolic internal dimension of $3.7 \mathrm{~cm}$, and concentric left ventricular hypertrophy (Figure 1A). Compared to a previous echocardiogram done months prior, the patient had a decline in LVEF (previously $60 \%$ ) when in atrial fibrillation. A coronary 


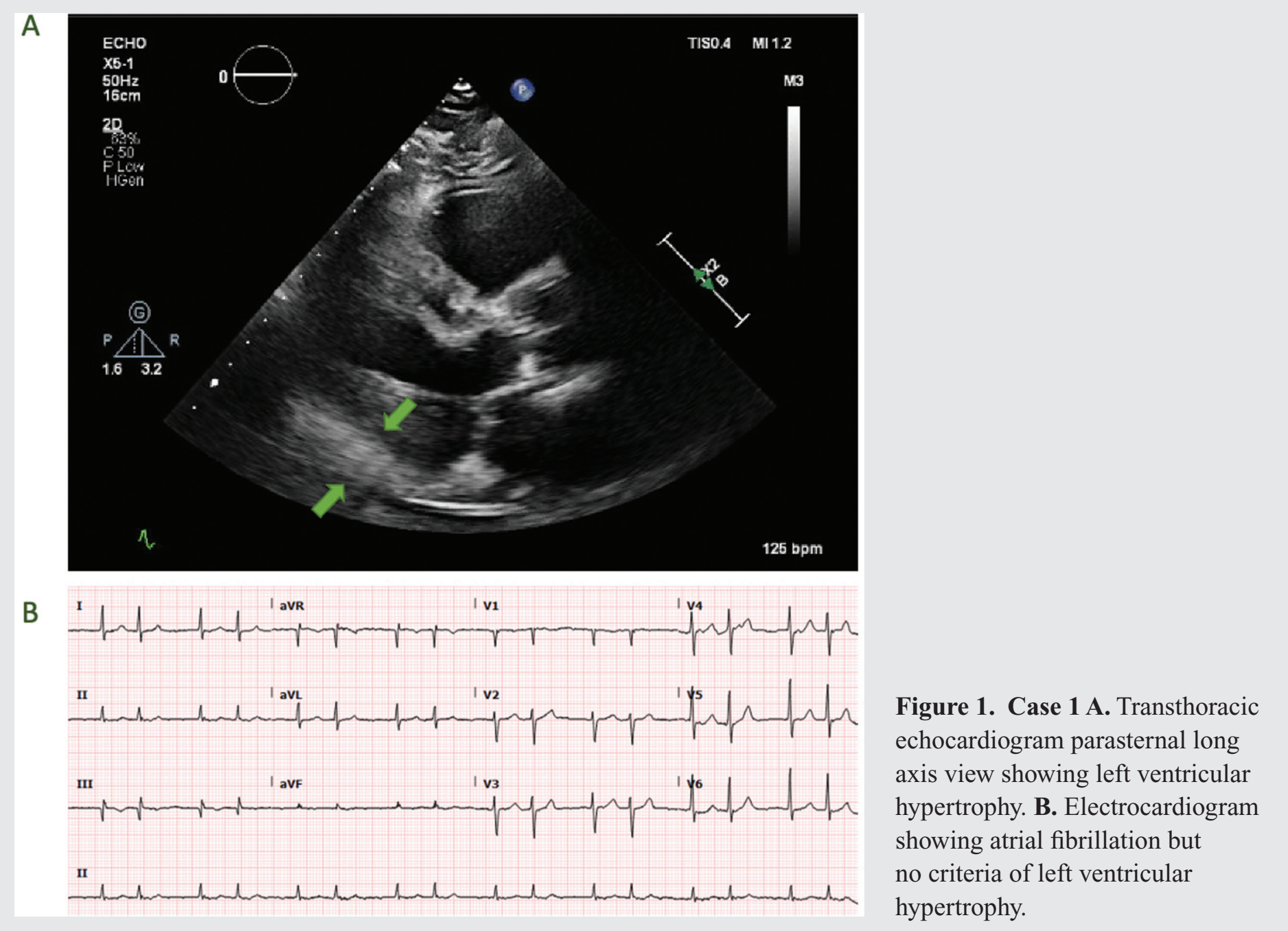

angiogram showed nonobstructive coronary artery disease. His ECG did not meet LVH criteria in the presence of concentric ventricular hypertrophy found on imaging (Figure 1B). He was not obese. Due to the suspicion for infiltrative causes of heart failure, testing for amyloid cardiomyopathy was started. The serum kappa/lambda free light chain ratio was normal; in addition, serum and urine electrophoresis with immunofixation was negative for monoclonal proteins. Tc99m pyrophosphate (PYP) scan was highly suggestive of ATTR-CM (Figure 2). No pathogenic variant of the TTR gene was found, and the patient was diagnosed with wATTR-CM with plans to start medical therapy with tafamidis, a transthyretin stabilizer that slows the formation amyloid.
Case 2: A 79-year-old man with hypertension and atrial fibrillation was being evaluated for unexplained dyspnea on exertion that had been increasing for 5 months. Dyspnea workup included pulmonary function tests that ruled out restrictive or obstructive pulmonary disorders, brain natriuretic peptide level that was $2,023 \mathrm{pg} / \mathrm{mL}$, and a TTE that demonstrated an LVEF of $61 \%$, left ventricular end-diastolic internal dimension of $3.7 \mathrm{~cm}$, concentric left ventricular hypertrophy (Figure 3A), and bi-atrial enlargement. Coronary angiography did not reveal significant coronary artery disease. An ECG showed rate-controlled atrial fibrillation without LVH criterion even in the presence of concentric ventricular hypertrophy found in the TTE (Figure 3B). Infiltrative causes of heart failure 

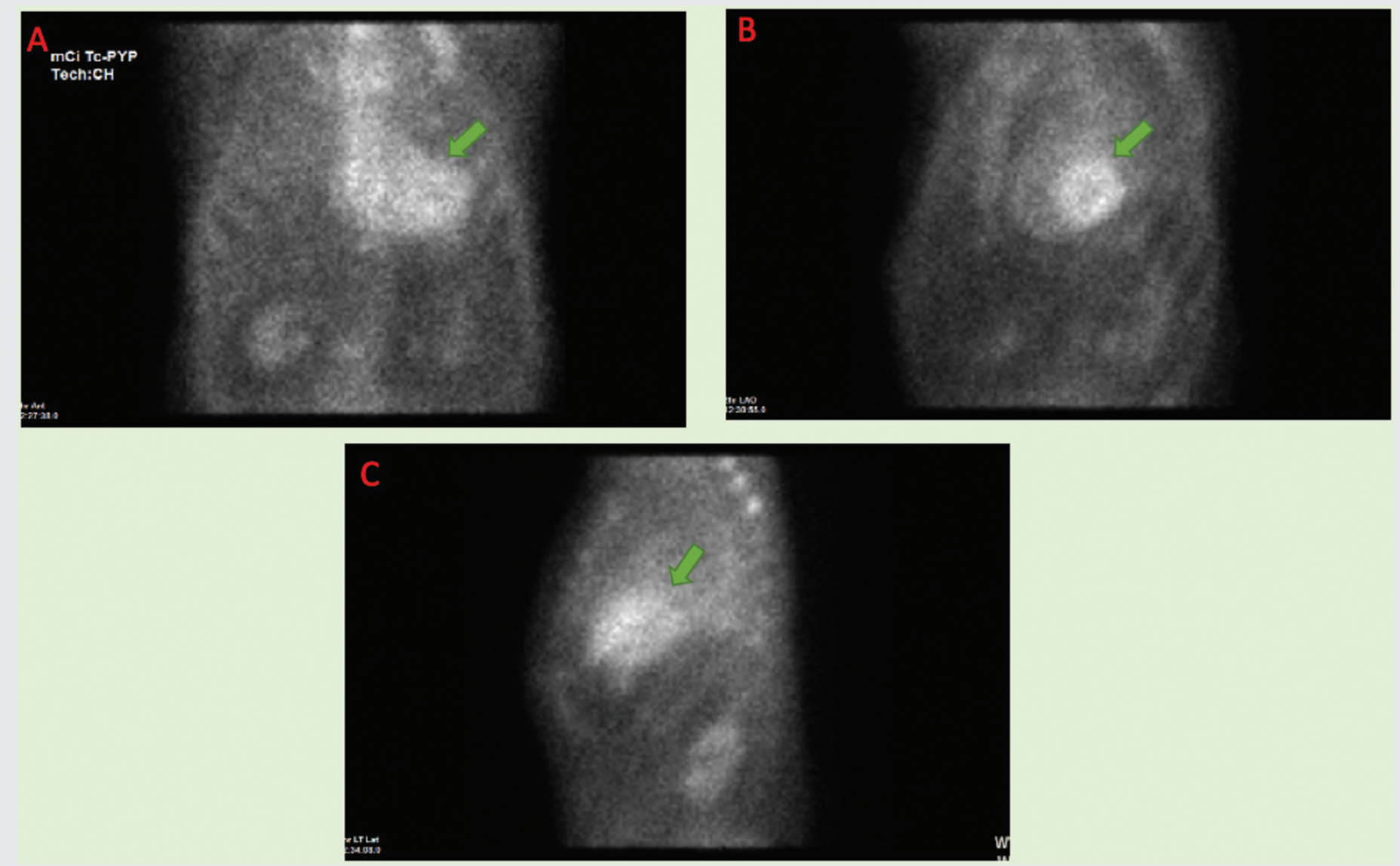

Figure 2. Case 1. Tc99m PYP scan. PYP scan with anterior (A) left anterior oblique (B) and lateral (C) view demonstrating Grade 3 update greater than rib uptake with mild/absent rib uptake. Heart to contralateral lung ratio 1.90.

were suspected. Primary amyloidosis was ruled out as described in Case 1, and a Tc99m PYP scan was strongly suggestive of ATTR-C. No pathogenic variant in the TTR gene was found. The patient was also diagnosed with wATTR-CM and scheduled to start medical treatment with tafamidis.

\section{Discussion}

Transthyretin amyloid cardiomyopathy is increasingly being recognized by cardiologists. Advances in noninvasive diagnosis and demonstration of the efficacy of specific ATTR-CM therapies ${ }^{6,7}$ have shifted ATTR-CM from a rarely suspected, encountered, and treated disease to a condition that physicians should consider in their differential diagnosis when evaluating patients with unknown causes of heart failure. We describe a diagnostic approach for ATTR-CM based on the most recent literature (Figure 4)

Transthyretin amyloid cardiomyopathy should be initially suspected in patients with heart failure (either with reduced or preserved ejection fraction) associated with any of the following: an increase in ventricular wall thickness without an obvious cause or disproportional to the history of hypertension, discordance between ventricular wall thickness and electrocardiographic voltage, age older than 65 years, especially males, and a history of central or peripheral nervous system involvement, such as carpal tunnel syndrome and spinal stenosis. After clinical suspicion 
A

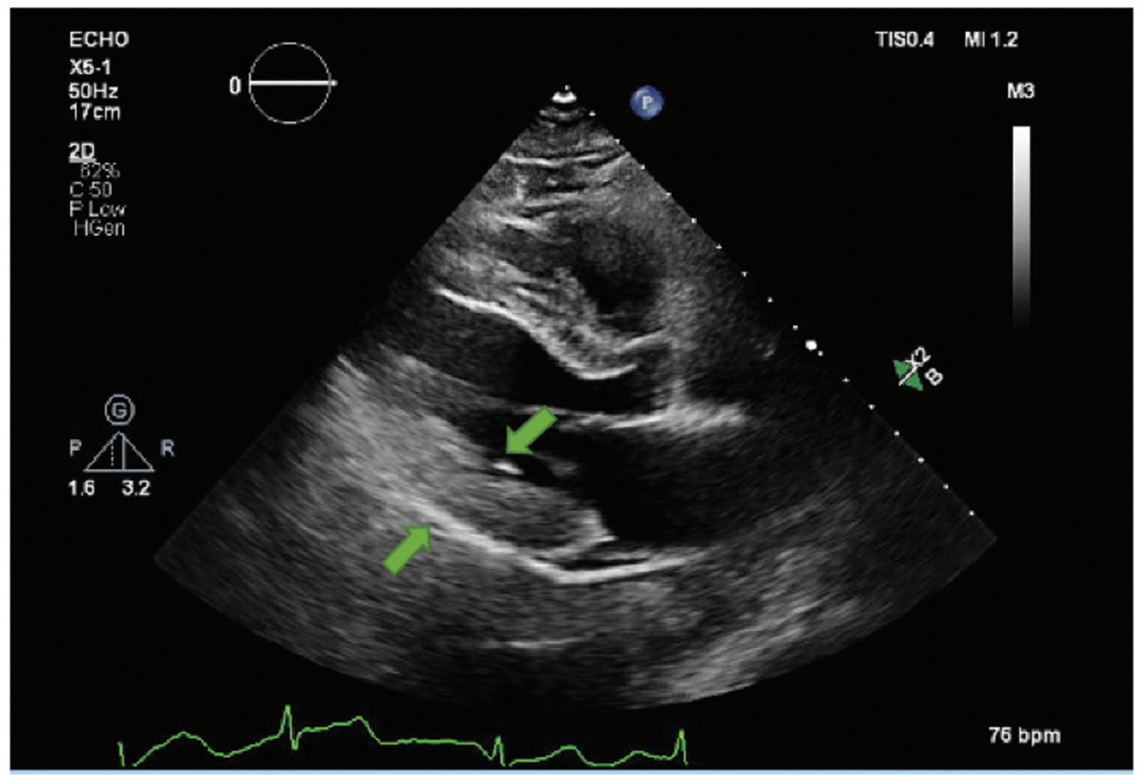

B

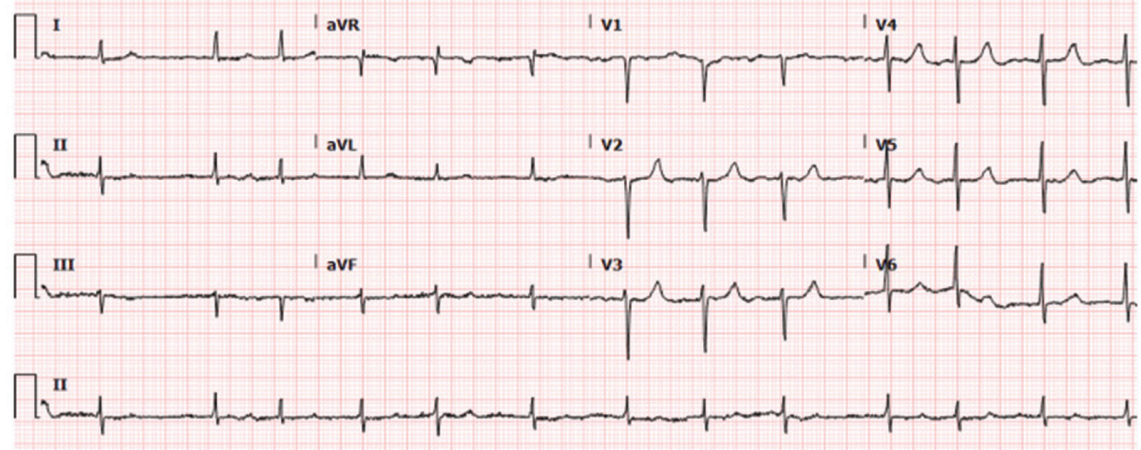

Figure 3. Case 2 A. Transthoracic echocardiogram parasternal long axis view showing left ventricular hypertrophy with speckled appearance. B. Electrocardiogram showing atrial fibrillation but no criteria of left ventricular hypertrophy.

of amyloid $C M$, the first step is to rule out $A L$ amyloidosis. This requires measurement of serum kappa/ lambda free light chain ratio and electrophoresis and immunofixation of serum and urine. In cases in which these tests are positive, the most likely etiology of the heart failure is systemic amyloidosis due to $A L$, and hematology evaluation is recommended.

Noninvasive diagnostic tests for ATTR-CM by nuclear scintigraphy using bone avid radiotracers, such as Tc-99m- PYP, Tc-99m 3,3-diphosphono1,2-propanodicarboxylic acid, or Tc-99m hydroxymethylene diphosphonate, are recommended when $A L$ amyloidosis has been ruled out. A positive test confirms the diagnosis of ATTR - CM. If there is a clinical suspicion of ATTR-CM in a patient with heart failure, nuclear scintigraphy using bone avid radiotracers have $100 \%$ specificity for ATTR-CM when grade 2 or 3 uptake is seen and there is an absence of a monoclonal protein in serum and urine testing. ${ }^{5}$ In the case of equivocal/non-diagnostic or negative tests but a high clinical suspicion, an endomyocardial biopsy has to be done with Congo red staining and identification of the precursor protein with mass spectroscopy or immunohistochemistry in myocardial tissue. If ATTR-CM is diagnosed, it is recommended to differentiate hereditary (hATTR-CM) from wild type (wATTR-CM). If a mutation is found, the patient is diagnosed with hATTR$\mathrm{CM}$; if not, the diagnosis is WATTR-CM.

The patients described above were suspected to have had WATTR-CM for a longer period before the 


\section{Unknown causes of heart failure + clinical suspicion of ATTR-CM*}

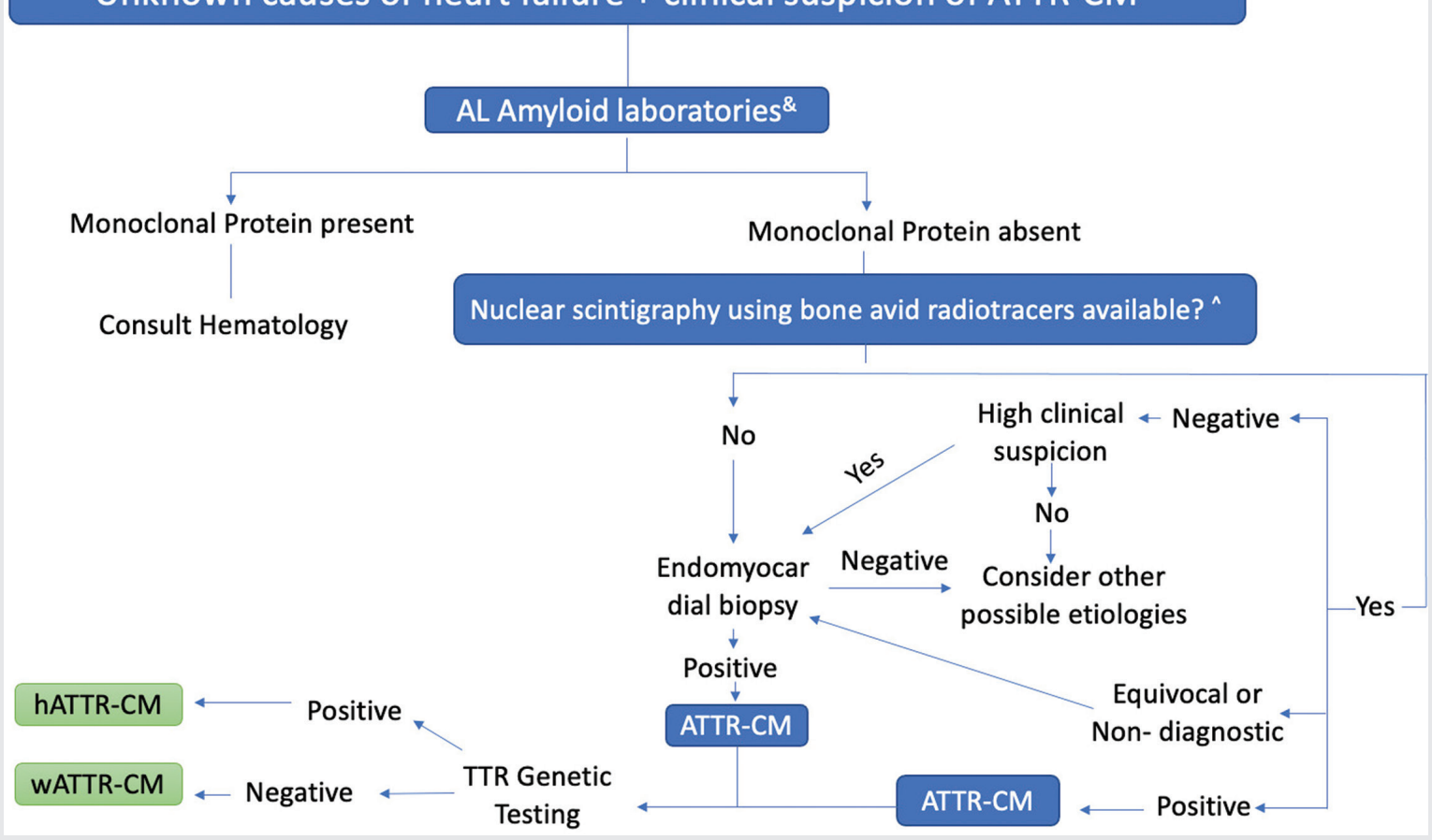

Figure 4. Proposed diagnostic algorithm.

* Suspicion for ATTR-CM: LVH/RVH, increased wall thickness without an obvious cause or disproportionate to the history of hypertension, discordance of wall thickness and electrocardiographic voltage, males older than 65 years, history of central or peripheral nervous system involvement (e.g., carpal tunnel syndrome, spinal stenosis).

\& Serum kappa/lambda free light chain ratio and immunofixation electrophoresis of serum and urine

Tc-99m- PYP (available in the United States), Tc-99m 3,3-diphosphono-1,2-propanodicarboxylic acid (DPD) or Tc-99m hydroxymethylene diphosphonate (HMDP),

AL: light chain, ATTR-CM: Transthyretin amyloid cardiomyopathy, hATTR-CM: hereditary amyloid cardiomyopathy, wATTR-CM: wild-type transthyretin cardiomyopathy.

final diagnosis was made, delaying the initiation of already approved therapies. ${ }^{7}$ The diagnosis depended on clinical suspicion, readily available diagnostic tests, and awareness of the disease.

\section{ConcLusion}

Advances in technology and treatment have shifted ATTR-CM from being a rarely suspected, encountered, and treated disease to a condition that physicians should consider in their differential diagnoses when evaluating patients with unknown causes of heart failure.
Article citation: Del Rio-Pertuz G, Jenkins LA, Sethi P, Argueta-Sosa E. Wild transthyretin amyloid cardiomyopathy recognition with noninvasive strategies: A case report and diagnostic approach for transthyretinrelated cardiomyopathy. The Southwest Respiratory and Critical Care Chronicles 2022;10(42):16-21

From: Department of Internal Medicine, Texas Tech University Health Sciences Center, Lubbock, Texas

Submitted: $12 / 13 / 2021$

Accepted: $1 / 10 / 2022$

Conflicts of interest: none

This work is licensed under a Creative Commons Attribution-ShareAlike 4.0 International License. 


\section{REFERENCES}

1. Wechalekar AD, Gillmore JD, Hawkins PN. Systemic amyloidosis. Lancet 2016;387(10038):2641-54.

2. Lioncino M, Monda E, Palmiero G, et al. Cardiovascular involvement in transthyretin cardiac amyloidosis. Heart Fail Clin 2022;18(1):73-87.

3. Ruberg FL, Grogan M, Hanna M, et al. Transthyretin amyloid cardiomyopathy: JACC State-of-the-Art Review. J Am Coll Cardiol 2019;73(22):2872-91.

4. Cruz Rodriguez JB, Tallaj JA. Narrative review of pharmacotherapy for transthyretin cardiac amyloid. Ann Transl Med 2021;9(6):519.
5. Gillmore JD, Maurer MS, Falk RH, et al. Nonbiopsy diagnosis of cardiac transthyretin amyloidosis. Circulation 2016; 133(24):2404-12.

6. Maurer MS, Schwartz JH, Gundapaneni B, et al. Tafamidis treatment for patients with transthyretin amyloid cardiomyopathy. N Engl J Med 2018;379(11):1007-16.

7. Griffin JM, Rosenthal JL, Grodin JL, et al. ATTR amyloidosis: current and emerging management strategies:. JACC CardioOncol 2021;3(4):488-505. 